edition an improvement on its predecessors. It is rarely that the turn of a phrase suggests that the text is a translation.

The book is concerned with the identification of fibres and the determination of the composition of blends of fibres. Until the start of the Second World War there were only five different chemical types of fibres although they occurred in many modifications. In the past few years many new chemical types have been introduced. In some cases they are being used for giving the handle and appearance of older and more expensive fibres, as the fibres which were once called "artificial silk" were used as substitutes for natural silk. In other cases complicated blends of fibres are being turned out with the purpose of combining the good qualities and of overcoming the bad qualities of individual fibres. In other cases again the new fibres are making available textile qualities not previously obtainable. All these changes have increased both the range and difficulty of the analytical problems facing the textile technologist. As a result much greater interest is now being taken in this field. Mr. Luniak has provided a guide; but, to continue the simile a little further, the extent of the field and the number of paths through it are increasing rapidly. This makes it difficult to write a book to include the very latest fibres and methods of analysis. Thus, for example, it has not been possible to include the chromatographic methods for identifying nylons.

A negligible prior knowledge has been assumed in the reader. Detailed instructions are given for carry. ing out the selected chemical, physical and microscopical tests. Any competent worker should be able to perform the first two immediately; but a considerable amount of practice is required before microscopical examinations can be done even with the clearest instructions. The value of the text is increased by the inclusion of appreciations of the tests, which are summarized in synoptic tables and schemes of analysis.

The book can be recommended particularly for its comprehensive coilection of excellent photomicrographs of natural and man-made fibres. These will be consulted by many readers who will not use the book as a laboratory manual.

J. M. Preston

\section{ABSTRACT SET THEORY}

\section{Abstract Set Theory}

By Prof. Abraham A. Fraenkel. (Studies in Logic and the Foundations of Mathematics.) Pp. xii +480 . (Amsterdam: North-Holland Publishing Co., 1953.) $38 f . ; 76 s$.

$\mathrm{M}$ ANY years ago the author published his "Einleitung in die Mengenlehre" (Springer, Berlin, 1919 ; third edition, 1928 ; reprinted Dover, New York, 1946). The present book follows, in external arrangement, the first half of the German treatise, but differs, not only through the principles introduced as basis of the theory, but also in the subject-matter and proofs in many sections. The definition by transfinite induction in $\$ 10,2$, and the direct proof of general comparability by means of the axiom of choice in $\$ 11,1$, are among subjects not treated in other text-books.

Chapter 1 is on elements and the concept of cardinal number. Most of this chapter, dealing with sets, denumerable sets, continuum, transcendental numbers, and cardinal numbers, could be read with great advantage by students reading for a mathematical honours degree.

Chapter 2 is concerned with equivalence and cardinals. The sections give definition of order, Cantor's theorem, the power set, the equivalence theorem, the problem of comparability, addition and multiplication of sets and of cardinals, inverse operations, inequalities between cardinals, the general concept of meet (inner product), Boolean algebra, exponentiation, infinitesimals and non-Archimedean domains.

Chapter 3 is on order and similarity, order types and ordinal numbers. There are sections on addition and multiplication of order types, linear sets of points, dense or continuous ordered sets, etc., well-ordered sets, transfinite induction, comparability of wellordered sets, ordinal numbers, comparability of ordinals, alephs and initial numbers, the well-ordering theorem, the general comparability of sets and cardinals.

A noteworthy feature of the book is the extremely extensive bibliography, which must have taken many years to prepare. This will be of great value to readers. 'The book is excellently written and produced, and should become a standard text-book on the subject.

R. G. COOKE

\section{TEACHING DEAF CHILDREN TO SPEAK}

Speech and the Deaf Child

By Irene R. Ewing and Dr. A. W. G. Ewing. Pp. xii $+256+4$ plates. (Manchester : Manchester University Press, 1954.) 18s. net.

7 TE chairman of the National College of Teachers of the Deaf recently summarized the present position in Great Britain as "approximately 5,000 deaf children in about fifty schools". These children are taught by some five hundred teachers, so that the educational provision made for them is comparable in size to one of the smaller local education authorities. They are educated by oral methods, speech and lip-reading forming the basis of the means of communication and, theoretically at least, every teacher of the deaf is a teacher of speech. The best teachers are usually too busy practising their craft to have time to record their methods for the benefit of their less successful colleagues, so it is very fortunate indeed that Dr. Irene Ewing, with the assistance of her husband, Prof. A. W. G. Ewing, has devoted the first years of her retirement to the production of this book. The Ewings form a unique team and are foremost among the workers in deaf education in Great Britain.

Although they begin by reviewing briefly the history of the teaching of speech to the deaf, they do not make sufficiently clear the confusion of methods that at present exists in the schools. Some teachers, using what is widely known as the 'analytic' method, still try to synthesize words and phrases from units of articulation; others who prefer the so-called 'synthetic' method teach the children to speak complete words or breath-groups of sounds, and correct mistakes by analysis. It is generally recognized that these methods, usually imposed upon the children, result in an artificial form of speech which, at its best, is both laboured and monotonous. This may be attributed to a certain extent to two main 